\title{
Experimental Approach for Assessing the Outcome Accuracy of Antibody Microarray Experiments
}

\author{
Qiang Gu ${ }^{*}$, Thamil Mani Sivanandam, and Jessica Haymore \\ Department of Neurobiology and Anatomy, Wake Forest University School of Medicine, Winston- \\ Salem, North Carolina 27157
}

\begin{abstract}
An experimental strategy for quality control of antibody microarray analyses is proposed. The method utilizes proteins that are prepared for regular antibody microarray experiments. There is no need to use exogenous positive or negative reference markers and no need to determine the absolute concentration of each individual protein in the sample. Validation experiments support the basic principle of the proposed approach. This method can be a useful tool for assessing the outcome accuracy of microarray experiments.
\end{abstract}

\section{Keywords}

microarrays; proteomics analysis; accuracy assessment; quality control

\section{Introduction}

Antibody microarray technology represents a powerful but still evolving tool for highthroughput proteomics analyses. ${ }^{1-7}$ The technique utilizes immobilized antibodies to capture target proteins of interest. A major advantage of the technique is that rather than comparing one or two proteins, one can examine a large number of proteins at a time. Because many physiological and pathological processes are complex in nature, by analyzing multiple proteins in parallel in the same sample, this technique can provide a broad overview of the magnitude and kinetics of changes in protein expressions that occur in normal and disease processes.

Because the antibody microarray analysis is a newly emerging technique, it remains critically important to develop quantitative means for quality control of the experimental outcome. The microarray technique involves multiple steps, from microarray production to the actual microarray experiment and subsequent data acquisition and analyses. Each step is critical in that it could potentially influence the accuracy of the experimental outcome. Although independent methods such as Western-blot and immunocytochemistry can be used to validate microarray results, they confirm microarray data only qualitatively, i.e., up- or down-regulation rather than the exact ratio of protein levels. In addition, it is impractical to apply these methods to confirm a large number of proteins one by one. Other approaches have been explored, for example, using labeled proteins with known concentrations as positive references in microarray experiments $8-10$ or using unlabeled proteins with known concentrations as negative references to displace labeled proteins in microarray experiments. ${ }^{2}$ These approaches, however, apply only to a limited number of targets with similar binding kinetics and are not suitable for validation of a large number of microarray spots that bind diverse targets with different binding

\footnotetext{
* To whom correspondence should be addressed. Dr. Qiang Gu, Department of Neurobiology and Anatomy, Wake Forest University School of Medicine, Medical Center Boulevard, Winston-Salem, North Carolina 27157; Phone, (336) 716-9226; Fax, (336) 716-4534; E-mail, qgu@wfubmc.edu.
} 
kinetics. Another approach is to test the same sample against itself to determine system errors, which also has limitations in that it can identify only potential false-positive but not falsenegative results. Thus, developing new approaches complementary to existing methods for assessing the accuracy of antibody microarray results will benefit future applications of the antibody microarray technology.

Here we describe an experimental approach that is aimed at determining whether the experimental condition and parameters are optimal and whether abundance ratios of proteins generated from regular antibody microarray experiments can accurately reflect the relative levels between two samples. To validate the basic principle of the proposed method, antibody microarray experiments were conducted using proteins extracted from the mouse visual cortex. The results support the validity of the proposed approach. These experiments also provide guidance and rationale for practical usage of this method.

\section{Experimental Section}

The procedure of antibody microarray experiments was similar as described previously. ${ }^{11} \mathrm{In}$ brief, animals were euthanized with pentobarbital. The visual cortex tissues were quickly dissected and frozen at $-20{ }^{\circ} \mathrm{C}$. Proteins were extracted using a protein extraction buffer (Clontech, Mountain View, CA). Tissue samples were homogenized with alumina ( $0.5 \mathrm{~g} / 100$ $\mathrm{mg}$ tissue) and extraction buffer ( $2 \mathrm{~mL} / 100 \mathrm{mg}$ tissue). The suspension was centrifuged at 10 $000 \times \mathrm{g}$ for $30 \mathrm{~min}$ at $4{ }^{\circ} \mathrm{C}$. The supernatant was collected, and its protein concentration was measured using BCA Protein Assay Kit (Pierce, Rockford, IL). The protein concentration was diluted to $1.1 \mathrm{mg} / \mathrm{mL}$ by adding an appropriate volume of the extraction buffer. Cy3 and Cy5 dyes (GE Healthcare, Piscataway, NJ) were dissolved in $110 \mu \mathrm{L}$ of extraction buffers, respectively. Then, $50 \mu \mathrm{L}$ of $\mathrm{Cy} 3$ and $50 \mu \mathrm{L}$ of $\mathrm{Cy} 5$ solutions were each mixed with $450 \mu \mathrm{L}$ protein solutions, respectively. After 90 min of incubation on ice, $4 \mu \mathrm{L}$ of $1 \mathrm{M}$ ethanolamine was added. After another 30 min of incubation, unbound dyes were removed by gel filtration using disposable PD-10 desalting columns (GE Healthcare). Each column was equilibrated with $3 \times 5 \mathrm{~mL}$ desalting buffer (Clontech) before adding a protein sample, which was eluted by applying $2 \mathrm{~mL}$ desalting buffer. The protein concentration in each sample was determined using BCA Protein Assay Kit (Pierce). Two antibody microarray incubation solutions were made with the following compositions: (1) $5 \mathrm{~mL}$ of incubation buffer (Clontech), $33.334 \mu \mathrm{g}$ of Cy3-labeled proteins, and $16.667 \mu \mathrm{g}$ of Cy5-labeled proteins, and (2) $5 \mathrm{~mL}$ of incubation buffer, $33.334 \mu \mathrm{g}$ of Cy5-labeled proteins, and $16.667 \mu \mathrm{g}$ of Cy3-labeled proteins. The homogeneity of the mixtures was achieved using a shaker with gentle rocking for $15 \mathrm{~min}$ at room temperature. Then the antibody microarray slides (Lot \# 6010064, Clontech) were added in the respective incubation chamber. After $30 \mathrm{~min}$ incubation at room temperature, the microarray slides were washed with seven different wash buffers (Clontech) at 5 min each, dried by centrifugation $(1000 \times \mathrm{g})$ in a swing bucket rotor at room temperature for $25 \mathrm{~min}$, and scanned using a confocal microarray scanner (ScanArray Gx) with a pixel resolution of $5 \mu \mathrm{m}$. The excitation wavelength and the emission filter wavelength for $\mathrm{Cy} 3$ and $\mathrm{Cy} 5$ were preset by the scanner's manufacturer (Perkin-Elmer, Shelton, CT) at $543 \mathrm{~nm} / 570 \mathrm{~nm}$ (excitation/ emission) and $633 \mathrm{~nm} / 670 \mathrm{~nm}$ (excitation/emission), respectively. The same strength of the laser power $(90 \%)$ and photomultiplier tube gain $(65 \%)$ were used for all scans of the microarray slides. Fluorescent signals of microarray spots were quantified using ScanArray Express (Perkin-Elmer).

\section{Results and Discussion}

The experimental design utilizes proteins that are prepared for regular antibody microarray experiments. The principle of the approach is shown in Figure 1. Two aliquots of the protein sample are made and labeled with Cy3 and Cy5, respectively. Microarray slide \#1 is incubated 
with a mixture containing $X$ amount of the Cy3-labeled proteins and $Y$ amount of the Cy5labeled proteins, whereas microarray slide \#2 is incubated with a mixture containing $X$ amount of the Cy5-labeled proteins and $Y$ amount of the Cy3-labeled proteins. A ratio analysis of a $\operatorname{target} T$ at spot $i\left(T_{i}\right)$ uses the formula:

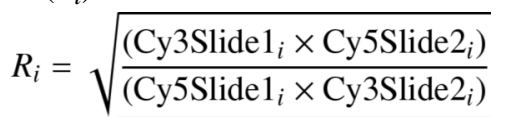

where $R_{i}=$ Ratio of $T_{i}$ between the Cy3- and Cy5-labeled proteins; Cy3Slide $1_{i}=$ Mean intensity of Cy 3 of spot $i$ on slide \#1 minus background; Cy5Slide $2_{i}=$ Mean intensity of Cy 5 of spot $i$ on slide \#2 minus background; Cy5Slide $1_{i}=$ Mean intensity of Cy5 of spot $i$ on slide \#1 minus background; and Cy3Slide $2{ }_{i}=$ Mean intensity of Cy 3 of spot $i$ on slide \#2 minus background.

Although it is not known how many different proteins exist in the sample, based on this design, for each existing protein in the sample the calculated ratio between Cy3-labeled and Cy5labeled proteins should be $X / Y$. Thus, the ratio $(X / Y)$ is universal for each protein on the microarray slide.

This formula can be easily explained using a simple example: when slide \#1 is incubated with $10 \mu \mathrm{g}$ of Cy3-labeled proteins $(X)$ and $5 \mu \mathrm{g}$ of Cy5-labeled proteins $(Y)$, while slide $\# 2$ is incubated with $10 \mu \mathrm{g}$ of Cy5-labeled proteins $(X)$ and $5 \mu \mathrm{g}$ of Cy3-labeled proteins $(Y)$, the theoretical ratio value for every protein should be:

$$
\left.R_{i}=\sqrt{\frac{(10 \times 10)}{(5 \times 5)}}=2 \text { (i.e., } \mathrm{X} / \mathrm{Y}\right)
$$

Experimental results of a protein at spot $n\left(T_{n}\right)$ with $R_{n}=2$ indicate that the experimental condition for $T_{n}$ is perfect and the experimentally obtained $T n$ ratio between the two samples is accurate. On the other hand, if the $R_{n}$ value is significantly different from the theoretic value, it would indicate that certain conditions or parameters of the experimental procedures are suboptimal and need adjustments to improve the accuracy of the experimental outcome.

To validate the basic principle of the proposed approach, antibody microarray experiments were performed using proteins extracted from the visual cortex of adult mice. A total of five experiments were repeated, which based on statistical power analysis achieved sufficient sample size for the validation. For each experiment, two aliquots of proteins were made and labeled with Cy3 and Cy5, respectively. Microarray slide \#1 was incubated with a mixture containing $33.334 \mu \mathrm{g}$ of Cy3-labeled proteins and $16.667 \mu \mathrm{g}$ of Cy5-labeled proteins, whereas microarray slide \#2 was incubated with a mixture containing $33.334 \mu \mathrm{g}$ of Cy5-labeled proteins and $16.667 \mu \mathrm{g}$ of Cy3-labeled proteins. Although the absolute concentration of each individual protein in the mixtures was unknown, the ratio of each individual $\mathrm{Cy} 3$ - and $\mathrm{Cy} 5$-labeled protein was constant, with 2:1 in incubation chamber \#1 and 1:2 in incubation chamber \#2. According to eq 1 , the theoretical outcome of the ratio measurement for each individual protein should be $R_{i}=2$.

The antibody microarrays used in this study had 1024 microarray spots on each slide. Among them, 6 spots were printed with fluorescence-labeled albumin as positive controls, whereas 4 spots were printed with nonlabeled albumin as negative controls. These 10 spots were excluded from the final data analyses. The ratio of each of the remaining 1014 spots was calculated using eq 1 and compared to the theoretical ratio $R_{i}=2$. The data showed an overall excellent match (Table 1). All 1014 spot ratios of the 5 experiments can be found in Supplementary Table S-1 (see Supporting Information). The results suggest that the experimental condition and parameters used in our experiments were optimal and that the antibody microarrays used in this study had high reproducibility. 
To show that background subtraction is an important step in eq 1, we calculated each ratio using the mean spot intensity with and without background subtraction (Table 1, Columns A and B). Statistical analyses were performed using ANOVA with Tukey HSD test to compare experimental data, both with and without background subtraction, to the theoretically expected value $R=2$. Data with or without background subtraction made a significant difference ( $p<$ $0.05)$. Although with background subtraction the outcome ratios $\left(R_{\text {group }}=1.981 \pm 0.036\right)$ were not significantly different from the theoretically expected ratio $R=2(p>0.05)$, the outcome ratios based on spot intensities without background subtraction $\left(R_{\text {group }}=1.837 \pm 0.053\right)$ were significantly different from the theoretically expected ratio $R=2(p<0.05)$. These results demonstrate that analyses with background subtraction provided more accurate results and that the background subtraction in eq 1 is a necessary step in this approach.

For conventional microarray data analyses, a normalization algorithm is often (but not always) applied to normalize spot intensities. The normalization methods are aimed at using specific algorithms to adjust data sets to remove systemic variations and allow each microarray to be adjusted individually. Because an artificial ratio is created in the proposed approach, conventional normalization methods could compute the difference of the two probes $(X / Y)$ as a systemic variation, e.g., a difference in the dye-labeling efficiency, and thus could correct the artificial ratio inappropriately. Therefore, it was expected that using conventional data normalization methods in the proposed formula could generate an inaccurate outcome. We applied a linear (TOTAL) and nonlinear (LOWESS) normalization algorithm, respectively. The linear algorithm determines a constant, based on the signal information of the entire array, to normalize all spots in the array field, whereas the nonlinear algorithm LOWESS (locally weighted scatter plot smoother) uses iterative fits to down weight points associated with outliers. 12,13 Both normalization methods generated overall ratios of about 1 compared to the theoretically expected ratio $R=2$ (Figure 2). These results suggest that conventional normalization algorithms may not be applicable in the proposed approach, which uses artificially created ratios that could be computed by conventional normalization algorithms as systemic variations. This is evidenced by the fact that the overall outcome with either of the two commonly used normalization methods yielded ratios close to 1 rather than the theoretically expected ratio 2 . Because the proposed eq 1 calculates combined spot information from two microarray slides with two colors on each slide and with dye-swap in each experiment, any systemic variations such as a difference of dye-labeling efficacy should be cancelled out or kept at a minimum. This notion is supported by the results that even without using any normalization method the experimental data showed an excellent outcome. Although the linear (TOTAL) and nonlinear (LOWESS) normalization methods could not help to improve the data accuracy in the validation experiments due to the artificially created ratio, they nevertheless demonstrated robust data correction ability in our experiments (Figure 2). Thus, new normalization algorithms or new variations of existing normalization algorithms are expected to be developed in the future that can be applied in conjunction with the proposed approach to further improve the accuracy of the experimental outcome.

Figure 3 shows microarray images of a validation experiment. It is evident that some microarray spots had very weak signals, indicating low abundance levels of the corresponding targets. We applied a commonly used threshold, defined as $2 \times \mathrm{SD}$ (standard deviation) of the local spot background, ${ }^{14-16}$ to filter out microarray spots that were either within the background noise range (spot mean < spot background $+2 \times \mathrm{SD}$ of the spot background) or within the saturation noise range (spot mean $>65536-2 \times \mathrm{SD}$ of the spot background). Statistical analyses of the group data either before or after spot filtering using the population ratios versus the theoretical ratio $R=2$ showed no significant difference between the experimental outcome and the theoretical value ( $n=5$ experiments, paired Student's $t$-test), with $p=0.624$ when all microarray spots are included and $p=0.987$ when the spot filtering is applied. However, spot filtering reduced the number of spots that generated large deviations from the theoretically expected 
ratio $R_{i}=2$ and, thus, reduced the overall variability (Figure 4) and improved the overall outcome (Table 1, Columns A and C).

Because each antibody was double printed side-by-side on the antibody microarray slides, we also determined the variability of each pair of the microarray spots using a threshold defined as $\log _{2}\left(R_{1} / R_{2}\right)=2 \times \mathrm{SD},{ }^{15,17}$ where $R_{1}$ and $R_{2}$ are the experimentally obtained ratios of spot \#1 and spot \#2, respectively, and SD is the standard deviation of the mean of $R_{1}$ and $R_{2}$. No spot pair showed $\log _{2}\left(R_{1} / R_{2}\right)>2 \times \mathrm{SD}$. Therefore, no spot was eliminated based on the spot variability threshold. 15,17 These results together demonstrated that the antibody microarrays used in the validation experiments had low background noise and consistent side-by-side spots.

Because both spot filtering and background subtraction improved the overall outcome, we proceeded to determine their impact individually. We compared the results between two groups: one with spot filtering and without background subtraction and the other with background subtraction and without spot filtering. Similar to the data with background subtraction, the data without background subtraction also showed improvements after spot filtering (Table 1, Columns B and D). The average ratio increased from $1.837 \pm 0.053$ to 1.892 \pm 0.047 , versus the theoretical ratio $R=2$. However, the group data with background subtraction and without spot filtering were still better than the group data without background subtraction but with spot filtering $(R=1.981 \pm 0.036$ versus $R=1.892 \pm 0.047)$. These data further support the importance of background subtraction and suggest that background subtraction has a stronger impact than spot filtering on the outcome accuracy in this approach.

The fact that data analyses with both background subtraction and spot filtering improved the overall data accuracy suggests that the proposed formula is not a stand-alone approach; rather, it has to be applied in conjunction with conventionally employed background subtraction and spot filtering methods. It is conceivable that spots with very low signal intensities could generate big errors because the noise level is proportionally higher in these spots. On the other hand, spots with very high signal intensities, which are close to the saturation range, could suppress the outcome ratio because some pixels within the spots could be already saturated when the mean intensity of the spot is not. Thus, conventional routines such as background subtraction and spot filtering are necessary in this approach.

The signal intensities of microarray spots can range widely from 1 to $65536\left(=2^{16}\right)$ in a standard 16-bit image. A universal formula should be applicable to all microarray spots regardless of whether their intensities are low, medium, or high. As indicated in Figure 4, data generated using eq 1 appear to be independent of the overall spot intensities. To validate this quantitatively, we conducted simple linear regression analyses using the overall intensity of the spots, defined as $I_{n}=$ Cy3Slide $1_{n}+$ Cy5Slide $2_{n}+$ Cy5Slide $1_{n}+$ Cy 3 Slide $2_{n}$ (because these four spot values are used in eq 1 for the ratio calculation), and the corresponding ratios $\left(R_{n}\right)$ to identify the line of best fit for each experiment (Table 2). The correlation coefficient values $\left(r^{2}\right)$ were small (Table 2), indicating no correlation between the output ratios and the overall spot intensities. We then tested the null hypothesis by comparing the slopes of the best-fitted lines with the theoretical noncorrelation slope $=0$ using paired Student's $t$-test. The $p$ values were 0.437 (all spots inclusive) and 0.656 (after spot filtering). Thus, the slopes of the bestfitted lines were not significantly different from 0 . These data confirm that the experimentally obtained ratios are dependent only on the relative intensities of the four spot values in eq 1 and independent of the overall spot intensities. Therefore, the proposed approach can be used for analyses of all spots, regardless of whether they have weak or strong signals.

The validation experiments used proteins extracted from mouse visual cortex. It is conceivable that proteins extracted from different brain regions, different organs, or different species may have different expression profiles. As shown in Table 1 (Column C), an average of 232 out of 
1014 spots was filtered out using a commonly used filtering threshold. This number could be attributed primarily to the low or non-expressed target proteins in the mouse visual cortex. Alternatively, some of the monoclonal antibodies on the microarray might recognize the target proteins of human or rat but not of mouse. However, despite the spot filtering, the valid spots (on average 782 spots in each experiment, $n=5$ experiments) remained powerful for statistical analyses, and for validating the proposed formula. Because the goal is to show proof-ofconcept, the absolute number of spots as well as the source of proteins should be less relevant in the validation experiments.

Although the validation experiments generated an overall excellent outcome, some spots showed higher variability of the results from slide to slide, for example spots \#29, \#30, \#155, \#156, \#171, \#172, \#611, and \#612 (Supplementary Table S-1). Two potential causes might contribute to such higher variability. One was due to very-low spot signal intensities, e.g., spots \#29, \#30, \#611, and \#612. Such low-intensity spots (representing low protein levels) could generate higher variability from slide to slide, because the signal-to-background ratio of these spots was low. Therefore, in practice, a threshold is often applied to filter out such spots.

Another cause appeared when some spots had irregular shapes, owing to imperfect microarray production, e.g., spots \#155, \#156, \#171, and \#172, which are indicated by arrows in Figure 3. When some slides had regular spots and others had irregular spots, higher variability from experiment to experiment could occur. Thus, a visual inspection of the scanned microarray images is necessary to identify and eliminate irregular spots.

Despite the fact that the averaged ratio of the group data $(R=1.999 \pm 0.036)$ fits well with the theoretical ratio $R=2$, there were a few valid spots $(4.3 \%)$ that generated compromised ratios that differed significantly from the theoretically expected ratio $R=2$. Because the majority of microarray spots in these validation experiments had an average ratio that was not significantly different from 2 and because the average is based on five experiments, it is unlikely that the difference of these few spots was caused by experimental errors. The most likely explanation could be that because dose-response curves of different antibodies may differ, the concentration of one or both of the Cy3-and Cy5-labeled proteins could be outside of the linear range of the antibody's dose-response curve, thereby generating an inaccurate ratio. If the artificial ratio used in the experiments $(X / Y=2: 1)$ was the defined differential expression threshold ( 2 fold), these spots could become false-positive if the ratio was significantly above 2 , or false-negative if the ratio was significantly below 2 . Thus, our approach could help to identify both potential false-positive and false-negative targets.

In fact, if different artificial ratios are created and corresponding antibody microarray experiments are performed, the results could be utilized as reference points to generate calibration curves. Because the results of such experiments could contain reference data for every protein on the slide, individual calibration curves for each protein could be generated based on a series of testing experiments. Such calibration curves could then be used to determine what the real ratio of a protein should be based on the results of regular antibody microarray experiments.

To demonstrate the usefulness of the proposed approach, we utilized this method in practice. Similar to the situation of data normalization where different algorithms exist, there are different quantitation methods for the calculation of spot intensities. For example, the software we have been using in our laboratory (ScanArray Express, Perkin-Elmer) offers 4 different quantitation algorithms: Adaptive Circle, Fixed Circle, Adaptive Threshold, and Histogram. 18 The questions we faced were whether these algorithms give the same outcome ratios, and if not, which one provides the most accurate experimental outcome. Because an artificial ratio $(X / Y=2)$ is created in our experiments, we know the theoretical outcome $R=2$. Thus, we could compare the experimental results generated using each of these algorithms. The quantitation 
using Adaptive Circle and Fixed Circle provided an overall accurate outcome, whereas that using Adaptive Threshold and Histogram generated a far less accurate outcome (Table 3). Statistic analyses further confirmed that the group data based on either Adaptive Circle or Fixed Circle are not significantly different from the theoretical ratio $R=2(p=0.713$ and $p=0.624$, respectively), whereas the group data based on Adaptive Threshold or Histogram are significantly different from $R=2$ ( $p=0.024$ and $p=0.001$, respectively). Because the experimental results using Fixed Circle generated a better mean group data and less variability (population ratios of each experiment range from 1.892 to 2.096 , SEM $=0.036$ ) than those using Adaptive Circle (population ratios of each experiment range from 1.876 to 2.317, SEM $=0.080$ ), we decided to use the Fixed Circle algorithm to calculate the spot intensities in our microarray analyses. This example demonstrates how the best algorithm was determined. Similarly, other critical parameters could be tested, and the optimal setting of each parameter could be identified. Such parameters could include, for example, different laser power strengths and different photomultiplier tube gains of the microarray scanner.

In conclusion, we described an experimental strategy for quality control of antibody microarray results. The validation experiments generated an excellent outcome when compared with the theoretical ratio and thus support the basic principle of the proposed approach. To utilize this approach in real experiments so that results of the testing experiments and regular experiments can be directly compared, we recommend that the regular antibody microarray experiments also use a two-color dye-swap design and that the total amount of proteins for the microarray incubation should be the same in both the testing and regular experiments. As long as the experimental parameters for the testing and regular antibody microarray experiments are the same, data generated from a few replicates of a single ratio test (e.g., $X / Y=$ differential threshold) could be used for the assessment of all regular antibody microarray experiments. Because all microarray technologies use the same principle, namely utilizing immobilized substrates at arrayed positions to capture targets of interest through affinity binding, the proposed strategy and formula should be applicable in general settings, regardless of whether DNA, oligonucleotide, protein, or antibody arrays are considered.

\section{Supplementary Material}

Refer to Web version on PubMed Central for supplementary material.

\section{Acknowledgements}

This work was supported by grants from NIH (EY14892) and The Mr. and Mrs. Tab Williams Jr. and Family Neuroscience Research and Program Development Endowment. Q.G. designed this approach, participated in the experiments and data analyses, and wrote the manuscript. T.S. participated in the microarray experiments and data analyses. J.H. participated in data analyses.

\section{References}

1. Haab BB. Proteomics 2003;3:2116-2122. [PubMed: 14595810]

2. Barry R, Soloviev M. Proteomics 2004;4:3717-3726. [PubMed: 15540209]

3. Perlee L, Christiansen J, Dondero R, Grimwade B, Lejnine S, Mullenix M, Shao W, Sorette M,

Tchernev V, Patel D, Kingsmore S. Proteome Sci 2004;2:9. [PubMed: 15598355]

4. Wingren C, Borrebaeck CA. Expert Rev Proteomics 2004;1:355-364. [PubMed: 15966831]

5. Angenendt P. Drug Discovery Today 2005;10:503-511. [PubMed: 15809196]

6. Eckel-Passow JE, Hoering A, Therneau TM, Ghobrial I. Cancer Res 2005;65:2985-2989. [PubMed: 15833819]

7. Kingsmore SF. Nat Rev Drug Discovery 2006;5:310-320.

8. Imbeaud S, Auffray C. Drug Discovery Today 2005;10:1175-1182. [PubMed: 16182210] 
9. Angenendt P, Glokler J, Murphy D, Lehrach H, Cahill DJ. Anal Biochem 2002;309:253-260. [PubMed: 12413459]

10. Andersson O, Kozlowski M, Garachtchenko T, Nikoloff C, Lew N, Litman DJ, Chaga G. J Proteome Res 2005;4:758-767. [PubMed: 15952723]

11. Gu Q, Sivanandam T, Kim CA. Proteome Sci 2006;4:21. [PubMed: 17034643]

12. Cleveland W. J Am Stat Assoc 1979;74:829-836.

13. Yang, YH.; Dudoit, S.; Luu, P.; Speed, TP. SPIE BiOS. San Jose, CA: 2001. http://stat-www.berkeley.edu/users/terry/zarray/html/normspie.html

14. Wang X, Ghosh S, Guo SW. Nucleic Acids Res 2001;29:E75. [PubMed: 11470890]

15. Yang IV, Chen E, Hasseman JP, Liang W, Frank BC, Wang S, Sharov V, Saeed AI, White J, Li J, Lee NH, Yeatman TJ, Quackenbush J. Genome Biol 2002;3:research0062.1-0062.13. [PubMed: 12429061]

16. Espina V, Mehta AI, Winters ME, Calvert V, Wulfkuhle J, Petricoin EF 3rd, Liotta LA. Proteomics 2003;3:2091-2100. [PubMed: 14595807]

17. Saama PM, Patel OV, Bettegowda A, Ireland JJ, Smith GW. Physiol Genomics 2006;28:62-66. [PubMed: 17003079]

18. PerkinEimer. ScanArray User Manual. 2004.

http://las.perkinelmer.com/content/Manuals/MAN_ScanArrayExpress20.pdf 


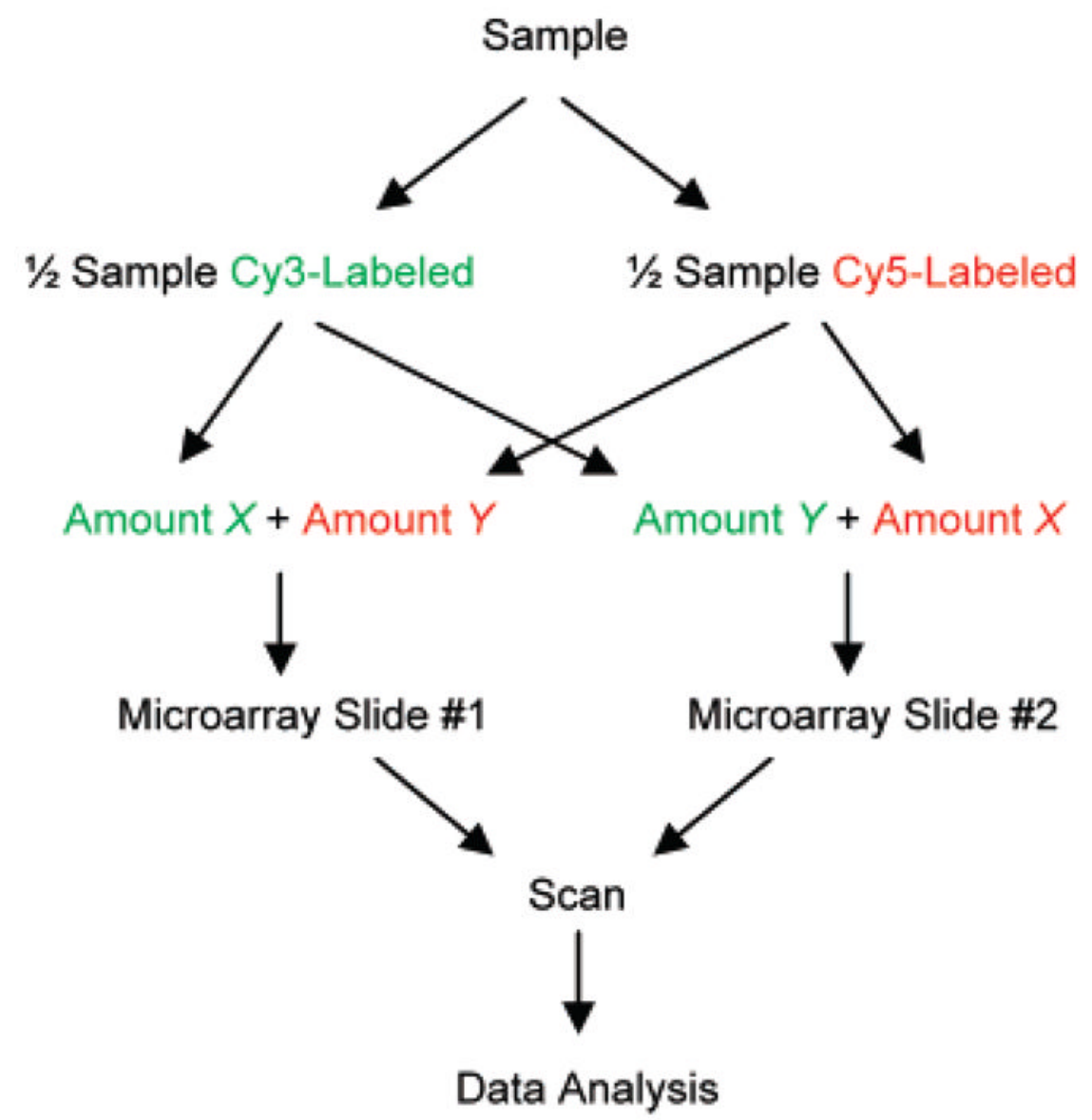

Figure 1.

Schematic illustration of the experimental principle. The design is based on a conventional two-color microarray experiment with dye-swap. Two modifications made this approach unique: (1) the probes are derived from one sample, and (2) the microarray slides are incubated with different amounts of different dye-labeled proteins. Different ratios of $X: Y$ will generate different experimental outcomes. 

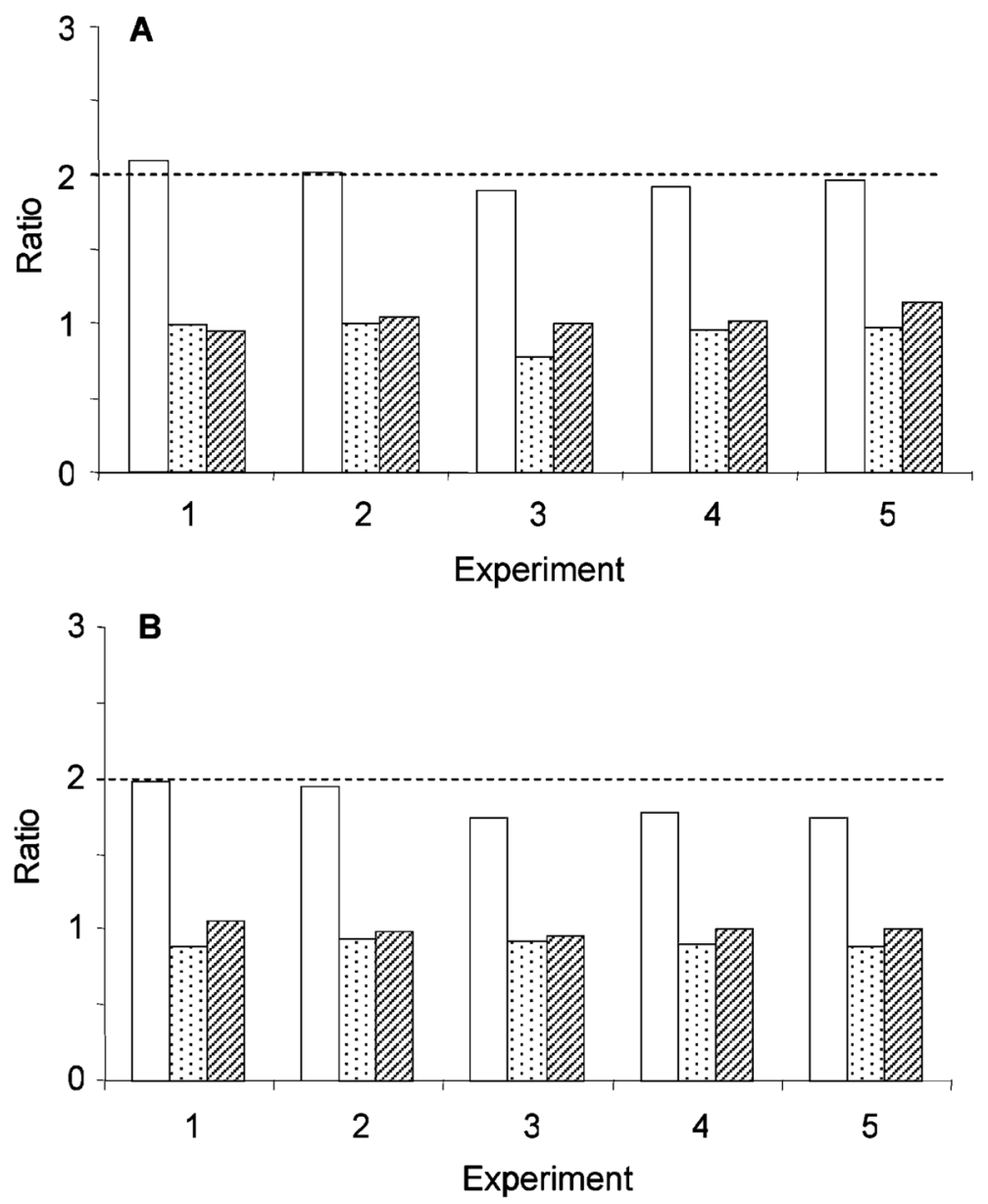

Figure 2.

Experimental results generated using spot intensities that were either unnormalized or normalized with TOTAL or LOWESS. The average of the population ratios of all 1014microarray spots of each experiment is shown. (A) Data with background subtraction. (B) Data without background subtraction. White columns, data without normalization; Dotted columns, data with TOTAL normalization; Lined columns, data with LOW-ESS normalization. The dotted line indicates the theoretically expected ratio of these experiments, $R=2$. 


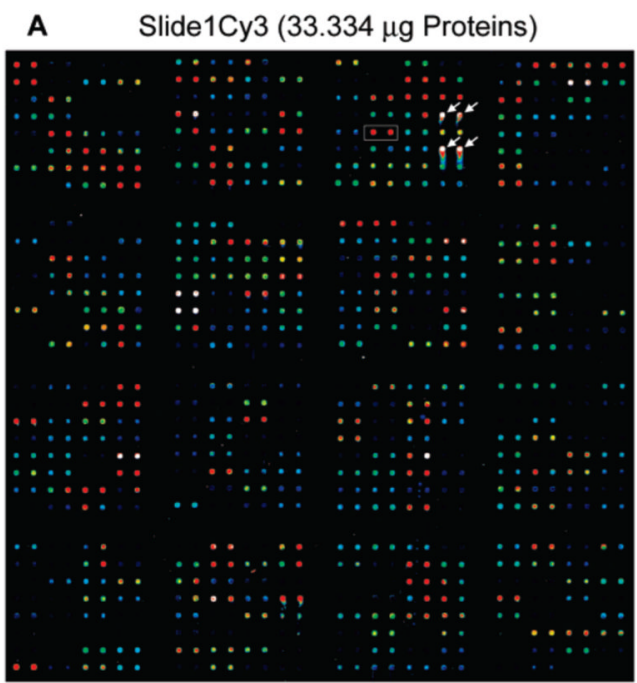

B $\quad$ Slide2Cy3 (16.667 $\mu$ g Proteins $)$

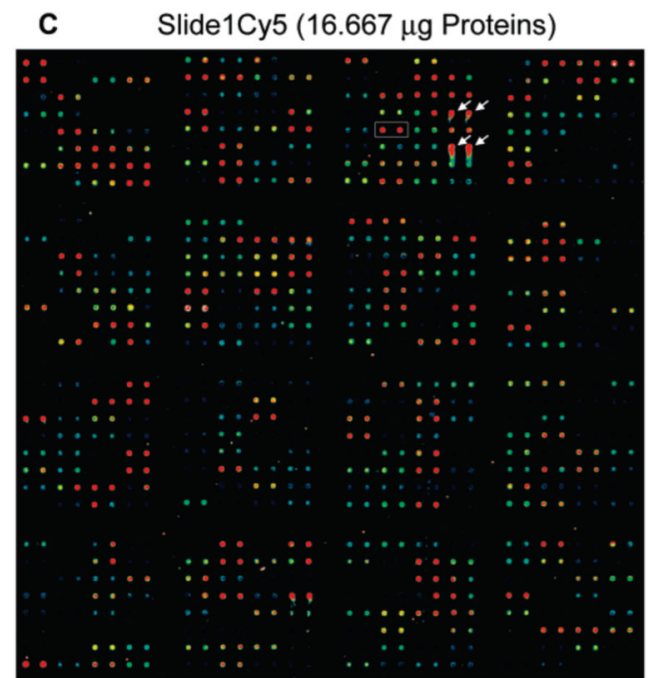

D

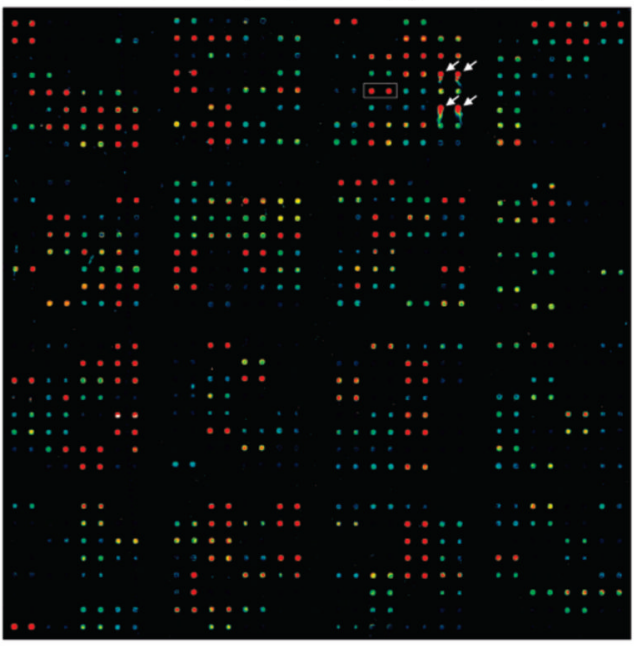

Slide2Cy5 (33.334 $\mu \mathrm{g}$ Proteins)

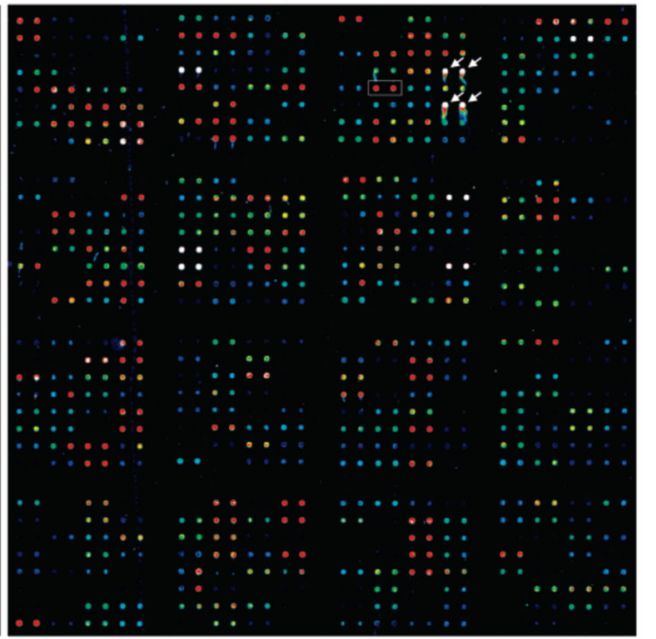

E Slide1Cy3

F $\quad$ Slide2Cy3
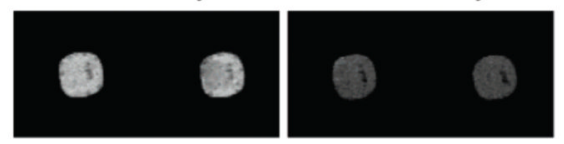

G Slide 1 Cy5

H Slide2Cy5

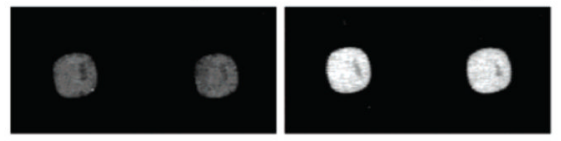

Figure 3.

(A-D) Microarray images with color-coded signal intensities of a validation experiment. Arrows point to four microarray spots with obvious irregular shapes. The rectangles indicate microarray spots of a representative protein (ubiquitin-conjugating enzyme E2L 3), which generated an average ratio $=2.00$ in this experiment, and are magnified in $\mathrm{E}-\mathrm{H}$ using a gray scale for better comparisons. 
Before Spot Filtering
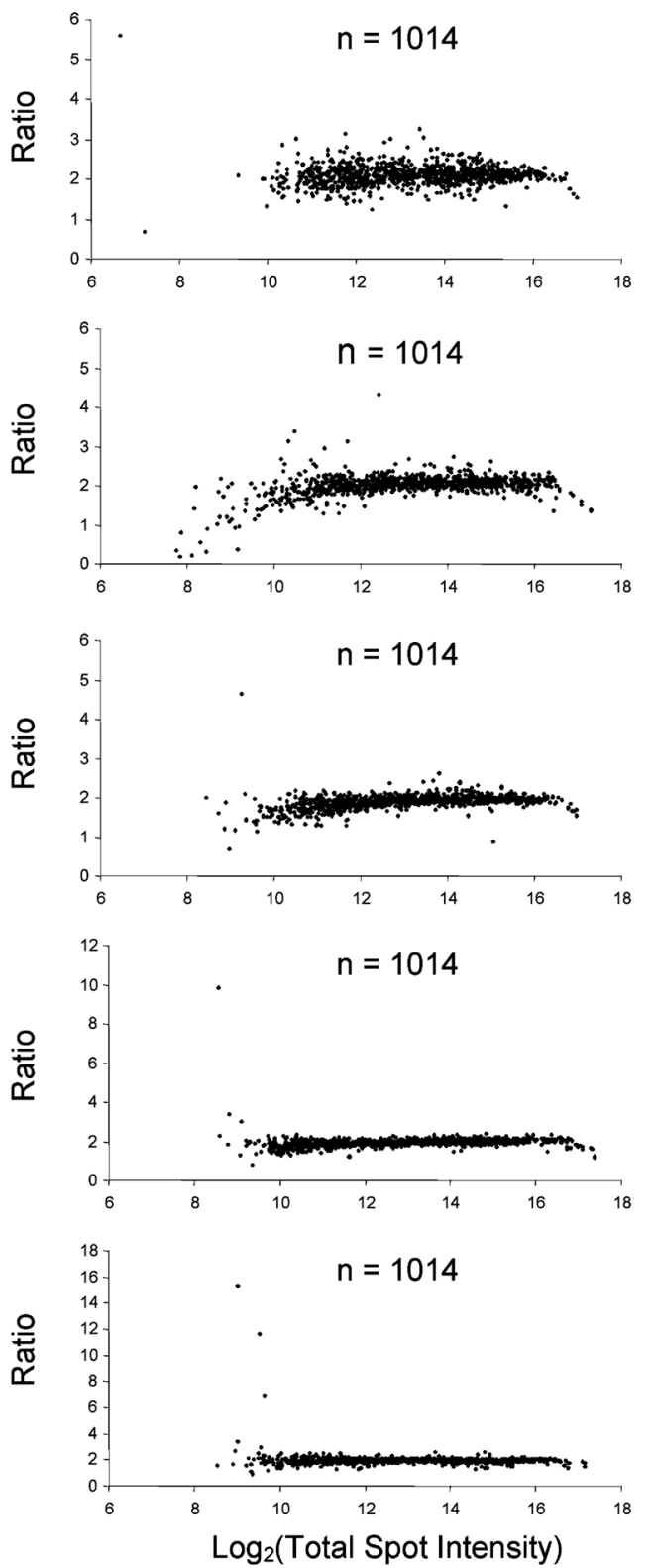

After Spot Filtering

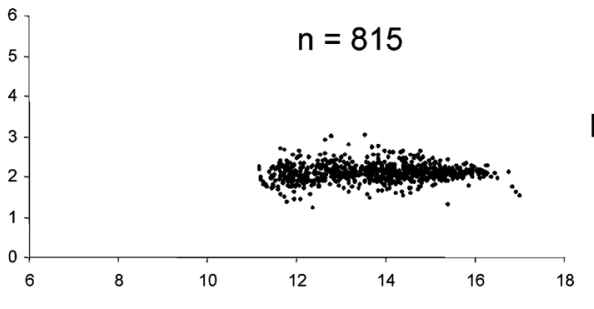

$\operatorname{Exp} \# 1$

Exp \#2

Exp \#3

$\operatorname{Exp} \# 4$

$\operatorname{Exp} \# 5$

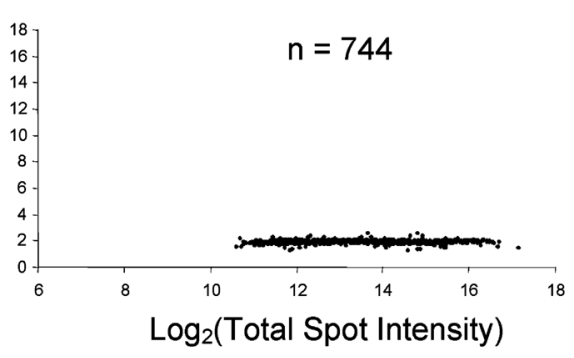

Figure 4.

Distribution patterns of the ratios of microarray spots before and after filtering. Because four spot values are used in eq 1 to generate the ratio of a target, the total spot intensity $(I)$ of each target $\left({ }_{i}\right)$ was defined as $I_{i}=$ Cy3Slide $1_{i}+$ Cy5Slide $2_{i}+$ Cy5Slide $1_{i}+$ Cy 3 Slide $2_{i}$. The ratio of each spot $\left(Y_{i}\right)$ was plotted against $\log 2\left(I_{i}\right)$. The cutoff threshold for spot filtering was $2 \times \mathrm{SD}$ of the local background. The theoretically expected ratio of these experiments was $R=2$. Note the changed ratio scales of experiments \#4 and \#5 due to a few spots with big deviations from $R=2$ before spot filtering and the elimination of such spots after spot filtering in all experiments. 
Table 1

Experimental Outcome Using Spot Intensities With (Columns A and C) and Without (Columns B and D) Background Subtraction, and Before (Columns A and B) and After (Columns C and D) Spot Filtering

\begin{tabular}{|c|c|c|c|c|}
\hline & $\mathbf{A}$ & B & C & D \\
\hline $\exp \#$ & $R_{\mathrm{m}-\mathrm{b}}$ (without filtering) & $R_{\mathrm{m}}$ (without filtering) & $R_{\mathrm{m}-\mathrm{b}}($ with filtering) & $R_{\mathrm{m}}$ (with filtering) \\
\hline $\begin{array}{l}1 \\
2 \\
3 \\
4 \\
5 \\
\text { group }\end{array}$ & $\begin{array}{l}2.096 \pm 0.008(n=1014) \\
2.023 \pm 0.008(n=1014) \\
1.892 \pm 0.006(n=1014) \\
1.931 \pm 0.010(n=1014) \\
1.963 \pm 0.018(n=1014) \\
1.981 \pm 0.036\end{array}$ & $\begin{array}{l}1.981 \pm 0.006(n=1014) \\
1.952 \pm 0.005(n=1014) \\
1.744 \pm 0.006(n=1014) \\
1.773 \pm 0.006(n=1014) \\
1.738 \pm 0.006(n=1014) \\
1.837 \pm 0.053\end{array}$ & $\begin{array}{l}2.106 \pm 0.007(n=815) \\
2.066 \pm 0.005(n=778) \\
1.931 \pm 0.005(n=766) \\
1.954 \pm 0.005(n=808) \\
1.940 \pm 0.005(n=744) \\
1.999 \pm 0.036\end{array}$ & $\begin{array}{l}2.017 \pm 0.007(n=815) \\
1.993 \pm 0.005(n=778) \\
1.814 \pm 0.005(n=766) \\
1.834 \pm 0.006(n=808) \\
1.800 \pm 0.006(n=744) \\
1.892 \pm 0.047\end{array}$ \\
\hline
\end{tabular}

${ }^{a} R_{\mathrm{m}-\mathrm{b}}$ and $R_{\mathrm{m}}$ are the average (Mean $\pm \mathrm{SEM}$ ) of the population ratios of the microarray spots with and without background subtraction, respectively. Spot filtering was conducted using $2 \times \mathrm{SD}$ of the local background as the cutoff threshold. $n$ indicates the number of microarray spots. 
Table 2

Simple Linear Regression of Experimental Results ${ }^{a}$

\begin{tabular}{|c|c|c|c|c|}
\hline \multirow[b]{2}{*}{$\exp \#$} & \multicolumn{2}{|c|}{ before spot filtering } & \multicolumn{2}{|c|}{ after spot filtering } \\
\hline & simple linear regression & $r^{2}$ & simple linear regression & $r^{2}$ \\
\hline 1 & $Y=2.0930334+0.0000002 X$ & 0.0002 & $Y=2.10455852-0.00000004 X$ & 0.00001 \\
\hline 2 & $Y=2.0085231+0.0000008 X$ & 0.0043 & $Y=2.0765804-0.0000005 X$ & 0.0047 \\
\hline 3 & $Y=1.8629542+0.0000018 X$ & 0.0345 & $Y=1.9164419+0.0000007 X$ & 0.0113 \\
\hline 4 & $Y=1.9139955+0.0000009 X$ & 0.0062 & $Y=1.9396157+0.0000007 X$ & 0.0155 \\
\hline 5 & $Y=1.9860097-0.0000014 X$ & 0.0028 & $Y=1.9458636-0.0000003 X$ & 0.0016 \\
\hline
\end{tabular}

${ }^{a}$ Best-fitted line for each validation experiment was determined by means of simple linear regression, using the outcome ratios $\left(Y_{i}\right)$ versus the total spot intensities $\left(X_{i}\right)$. The total spot intensity for each spot $(i)$ was defined as $I_{i}=\mathrm{Cy} 3 \operatorname{Slide} 1_{i}+\mathrm{Cy} 5 \mathrm{Slide} 2 i+\mathrm{Cy} 5 \mathrm{Slide} 1_{i}+\mathrm{Cy} 3 \mathrm{Slide} 2 i$. The value $r^{2}$ is the square of the Pearson product-moment correlation coefficient. 
Table 3

Experimental Outcome Using Different Quantitation Algorithms for the Determination of Spot Intensities ${ }^{a}$

\begin{tabular}{|c|c|c|c|c|}
\hline $\exp \#$ & $\boldsymbol{R}$ (Adaptive Circle) & $R$ (Fixed Circle) & $R$ (Adaptive Threshold) & $R$ (Histogram) \\
\hline $\begin{array}{l}1 \\
2 \\
3 \\
4 \\
5 \\
\text { group }\end{array}$ & $\begin{array}{l}2.066 \pm 0.007 \\
2.002 \pm 0.007 \\
1.876 \pm 0.005 \\
1.896 \pm 0.005 \\
2.317 \pm 0.008 \\
2.031 \pm 0.080\end{array}$ & $\begin{array}{l}2.096 \pm 0.008 \\
2.023 \pm 0.008 \\
1.892 \pm 0.006 \\
1.931 \pm 0.010 \\
1.963 \pm 0.018 \\
1.981 \pm 0.036\end{array}$ & $\begin{array}{l}1.974 \pm 0.007 \\
1.934 \pm 0.006 \\
1.788 \pm 0.005 \\
1.805 \pm 0.006 \\
1.788 \pm 0.006 \\
1.858 \pm 0.040\end{array}$ & $\begin{array}{l}1.695 \pm 0.007 \\
1.733 \pm 0.008 \\
1.537 \pm 0.007 \\
1.586 \pm 0.009 \\
1.495 \pm 0.007 \\
1.609 \pm 0.046\end{array}$ \\
\hline
\end{tabular}

$a_{R}$ is the average (Mean $\left.\pm \mathrm{SEM}\right)$ of the population ratios of the microarray spots $(n=1014)$. The theoretically expected outcome was $R=2$. 